\title{
Continuous-Flow Electrophilic Amination of Arenes and Schmidt Reaction of Carboxylic Acids Utilizing the Superacidic Trimethylsilyl Azide/Triflic Acid Reagent System
}

\author{
Yuesu Chen, ${ }^{\dagger}$ Bernhard Gutmann, ${ }^{\ddagger}$ and C. Oliver Kappe*, ${ }^{\dagger}$ \\ ${ }^{\dagger}$ Institute of Chemistry, University of Graz, NAWI Graz, Heinrichstrasse 28, A-8010 Graz, Austria \\ ${ }^{\ddagger}$ Research Center Pharmaceutical Engineering GmbH (RCPE), Inffeldgasse 13, 8010 Graz, Austria \\ * C. Oliver Kappe. E-mail: oliver.kappe@uni-graz.at.
}

\section{Table of Contents:}

1. Flow Setup: Construction and Maintenance...........................................S2

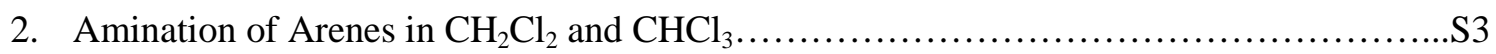

3. Amination Control Experiments without Azide..................................... 3

4. Optimization Data for Schmidt Reaction................................................ 4

5. NMR Spectra of All Isolated Products.........................................S4-S17 


\section{Flow Setup: Construction and Maintenance}

Pumps (P1, P2 and P3 for stream 1, 2 and 3): Syrris Asia 3.0. Loops (L1 and $\mathbf{L 2}$ for stream 1 and 2) are used for the storage of reactants. Their volumes can be varied to fit the desired reaction scale (Syrris). Reaction coils (R1 and R2): R1 (PTFE, o.d. 1/16 in, i.d. $0.8 \mathrm{~mm}, 6.0 \mathrm{~mL}$ ) is used for amination or Schmidt reaction; R2 (PTFE, o.d. 1/16 in, i.d. $0.8 \mathrm{~mm}, 5.0 \mathrm{~mL}$ ) for quenching. Mixers: M1 (Tefzel, ETFE cross assembly, o.d. 1/16 in, i.d. $0.5 \mathrm{~mm}$ ) and M2 (Tefzel, ETFE tee assembly, o.d. 1/16 in, i.d. $0.5 \mathrm{~mm}$ ). Back pressure regulator BPR (Upchurch Scientific): adjusted to 7 bar. Maintenance: The whole setup should be flushed thoroughly with methanol or isopropanol after each run. The reaction can only be started when (1) the whole setup has been flushed with $\mathrm{CHCl}_{3}$ (P1 and P2) and $\mathrm{MeOH}-\mathrm{H}_{2} \mathrm{O}(\mathrm{P} 3)$ thoroughly and (2) the temperature of the oil bath has stabilized.

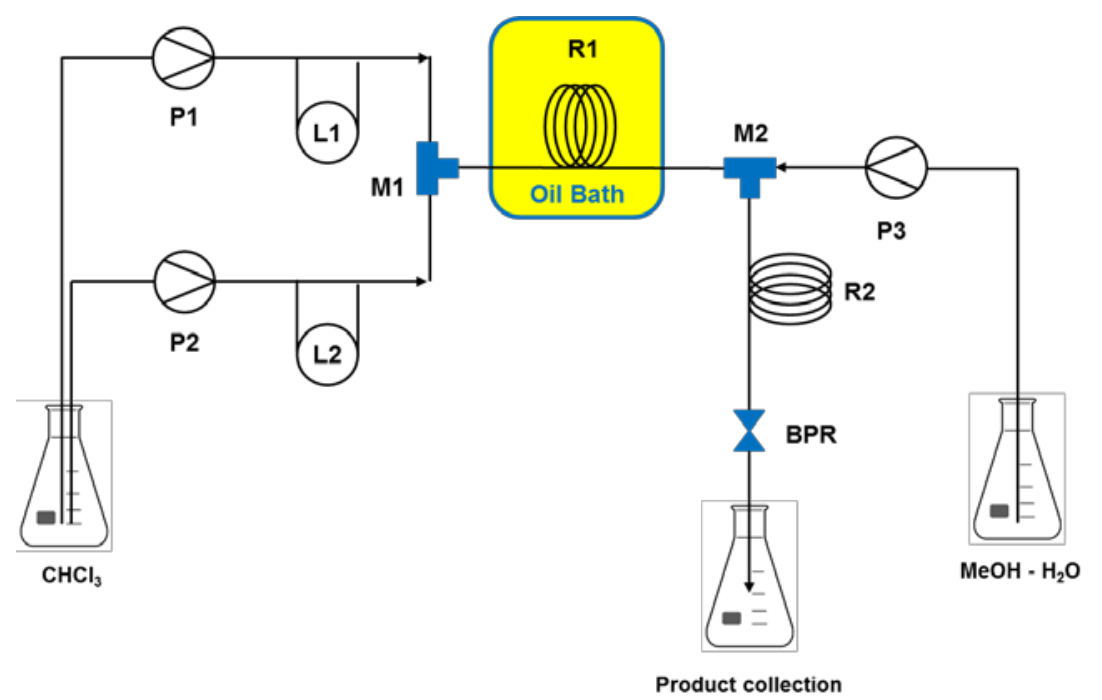

Figure S1. Layout of the Flow Setup

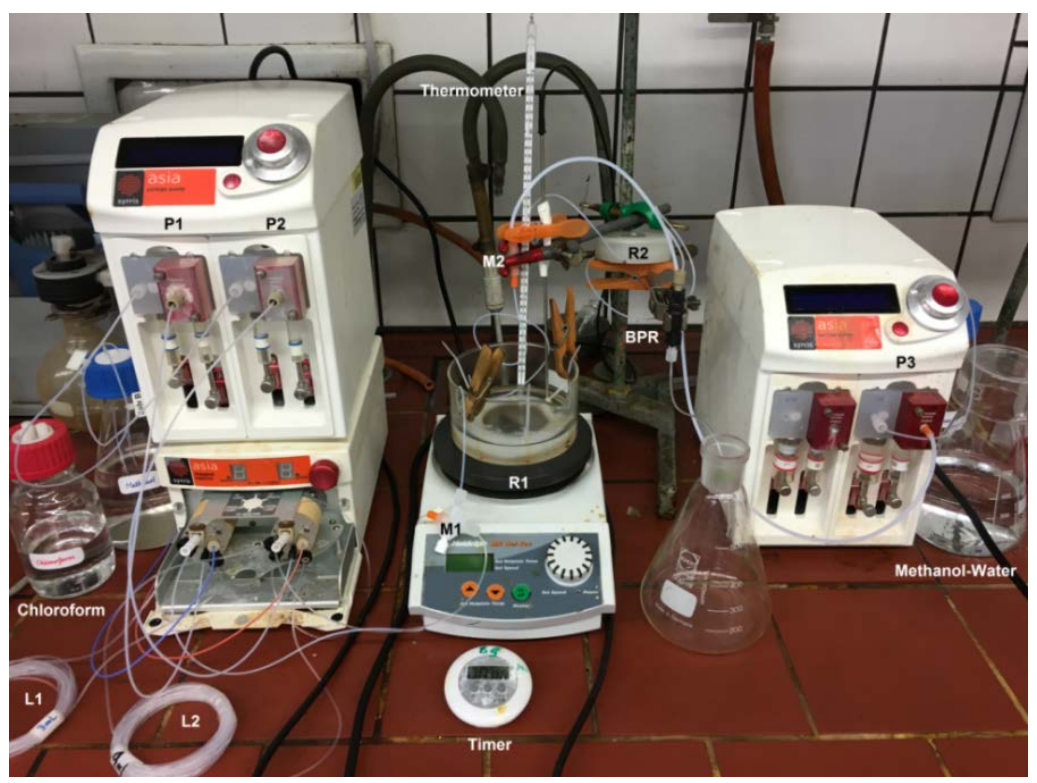

Figure S2. Image of the flow setup. 
Scheme S1. A Proposed Mechanism for the Formation of Chlorotoluidines

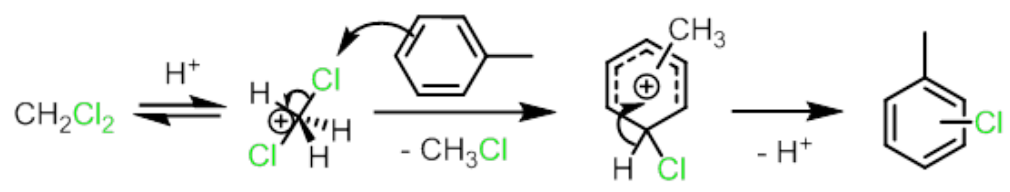
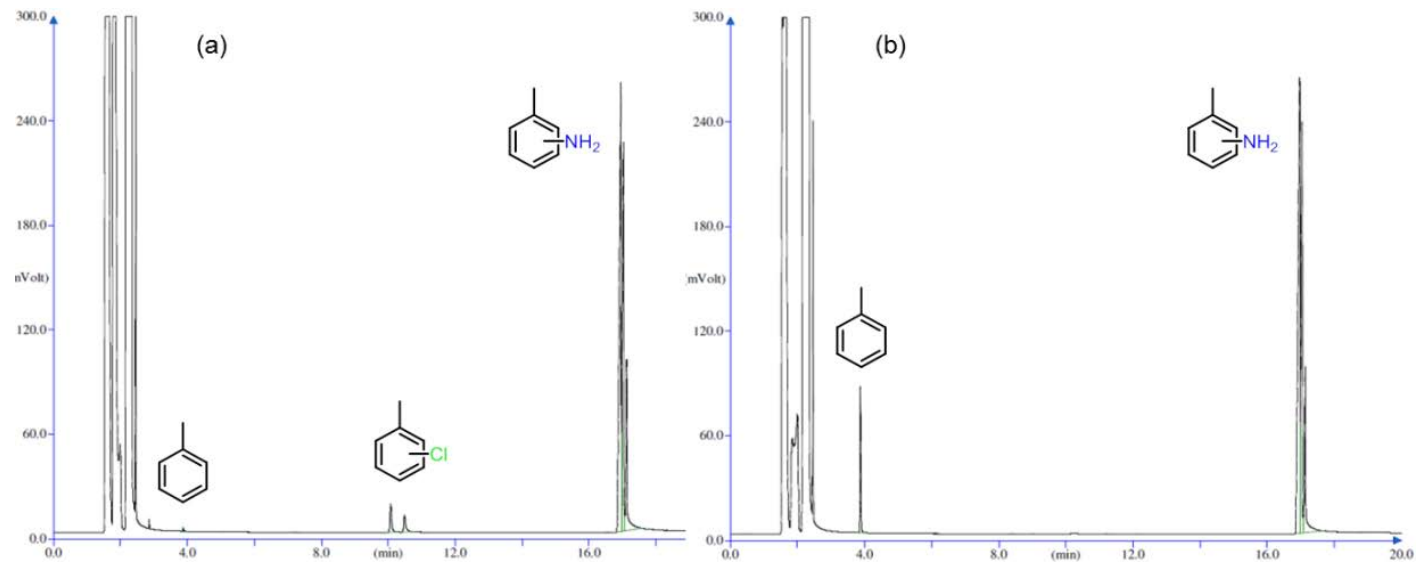

Figure S3. Gas chromatograms of the mixtures using (a) dichloromethane and (b) chloroform as solvent.
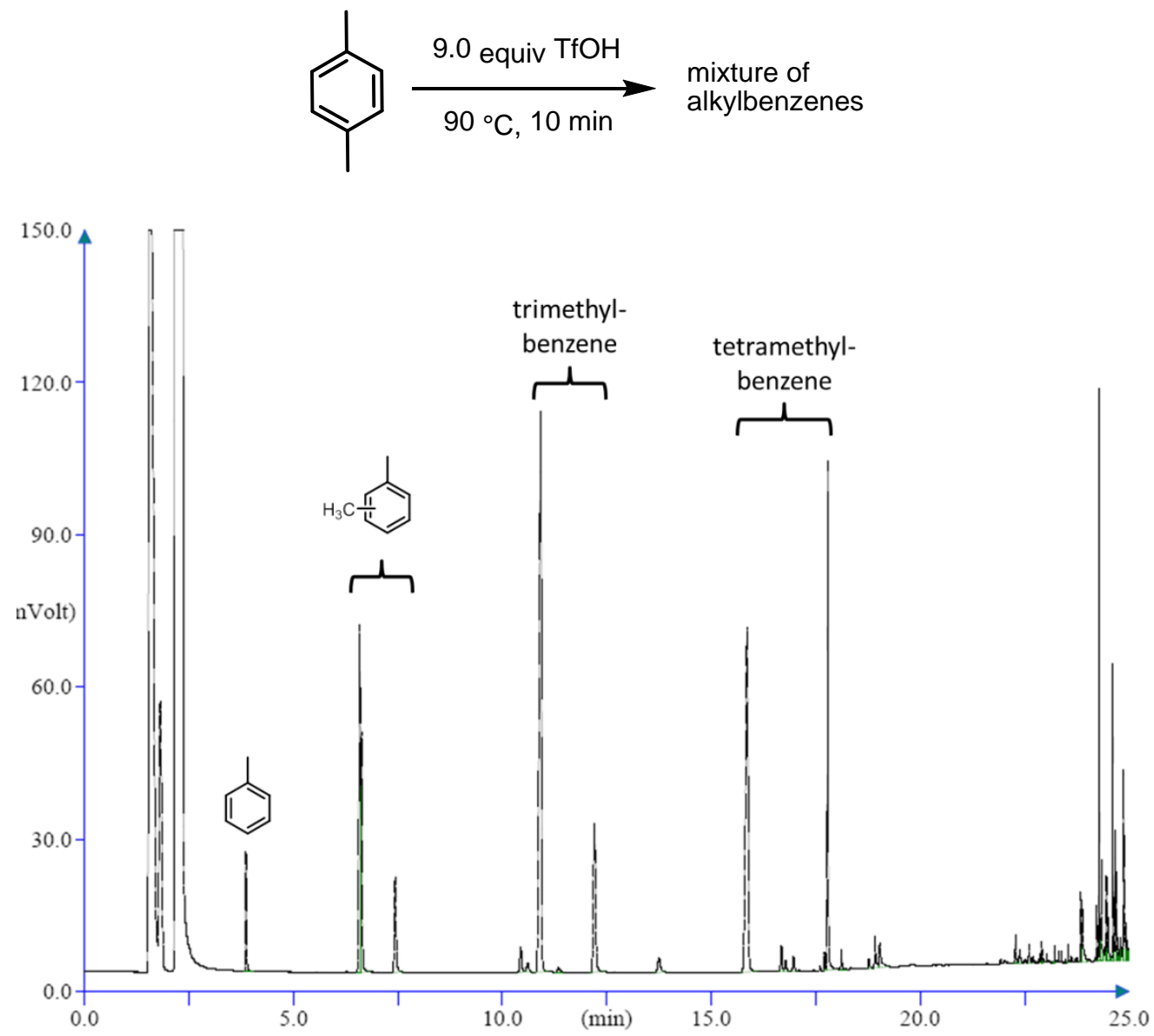

Figure S4. Control experiment without $\mathrm{TMSN}_{3}$ (entry 3, Table 6 without $\mathrm{TMSN}_{3}$ ). 
Table S1. Catalyst Screening for Schmidt Reaction of 4-Chlorobenzoic Acid

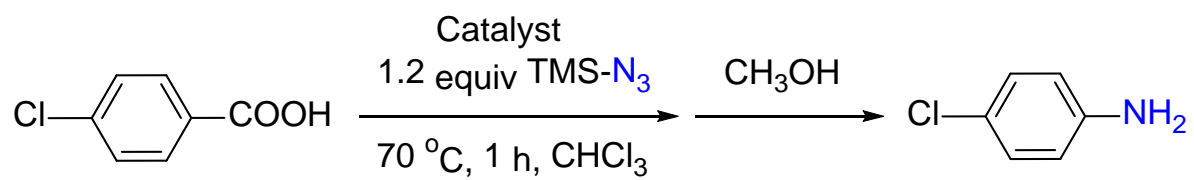

\begin{tabular}{cccc}
\hline entry & catalyst & yield $^{a}(\%)$ & remarks \\
\hline 1 & conc. $\mathrm{H}_{2} \mathrm{SO}_{4}(9.0$ equiv) & 75 & $\begin{array}{c}\text { ArCOOH not soluble } \\
\text { dissolves ArCOOH at } \mathrm{rt}\end{array}$ \\
2 & Fuming $\mathrm{H}_{2} \mathrm{SO}_{4}\left(20 \% \mathrm{SO}_{3}\right)$ & 82 & \\
3 & $\mathrm{TfOH}$ (4.5 equiv) & 0 & \\
4 & TfOHeral byproducts detected & \\
5 & TfOH (4.5 equiv) + TFA (4.5 equiv) & 21 & \\
6 & TfOH (9.0 equiv) & 27 & dissolves ArCOOH at rt \\
\hline
\end{tabular}

${ }^{a}$ 4-Chlorobenzoic acid (0.2 mmol); yields were calculated from HPLC peak area using an external standard; ${ }^{b}$ The reaction mixtures were quenched with methanol for HPLC and LC-MS analysis

NMR-Spectra of Products $\left({ }^{1} \mathrm{H} 300 \mathrm{MHz},{ }^{13} \mathrm{C} 75 \mathrm{MHz}\right.$, Solvent: $\mathrm{CDCl}_{3}$ )

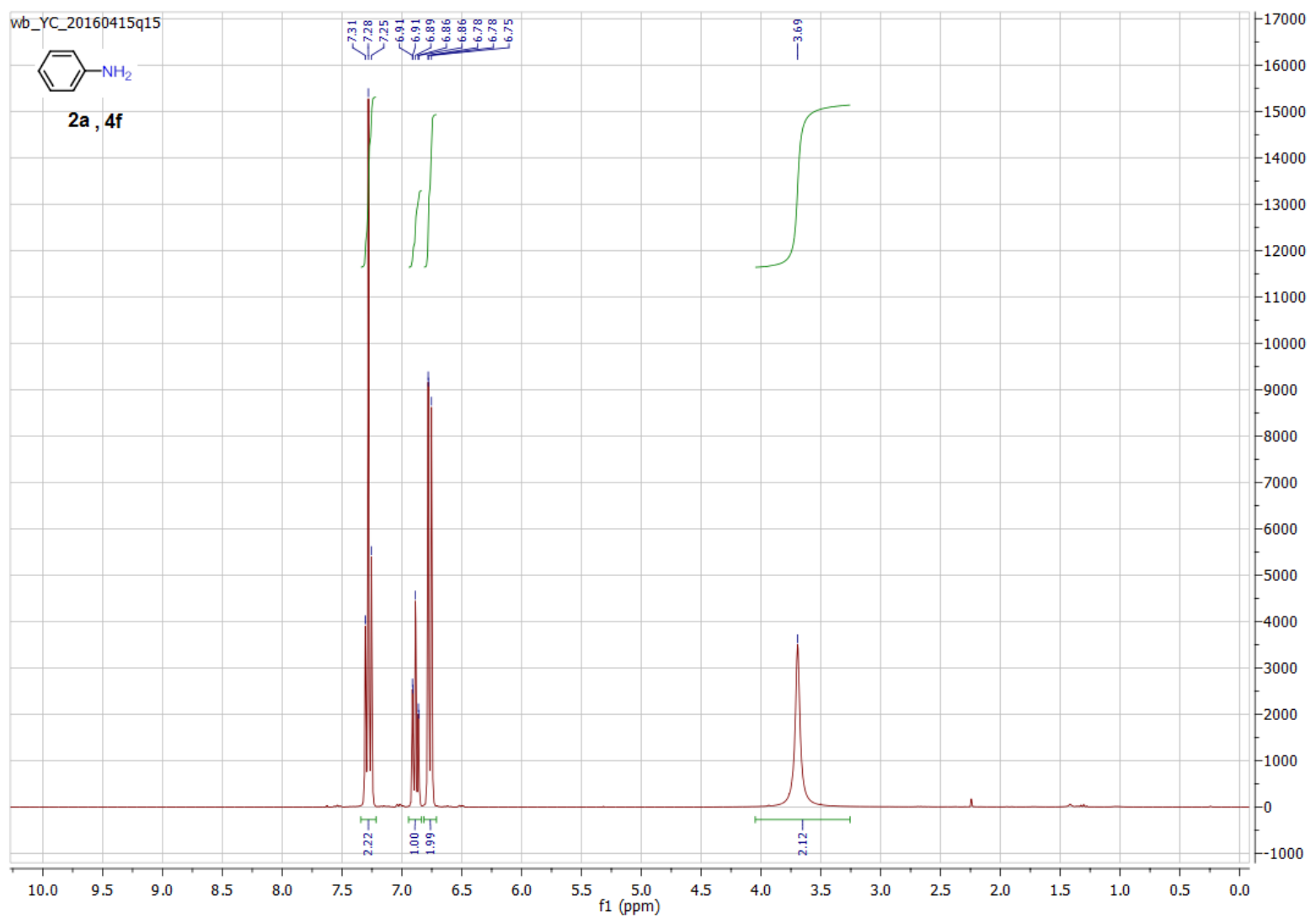



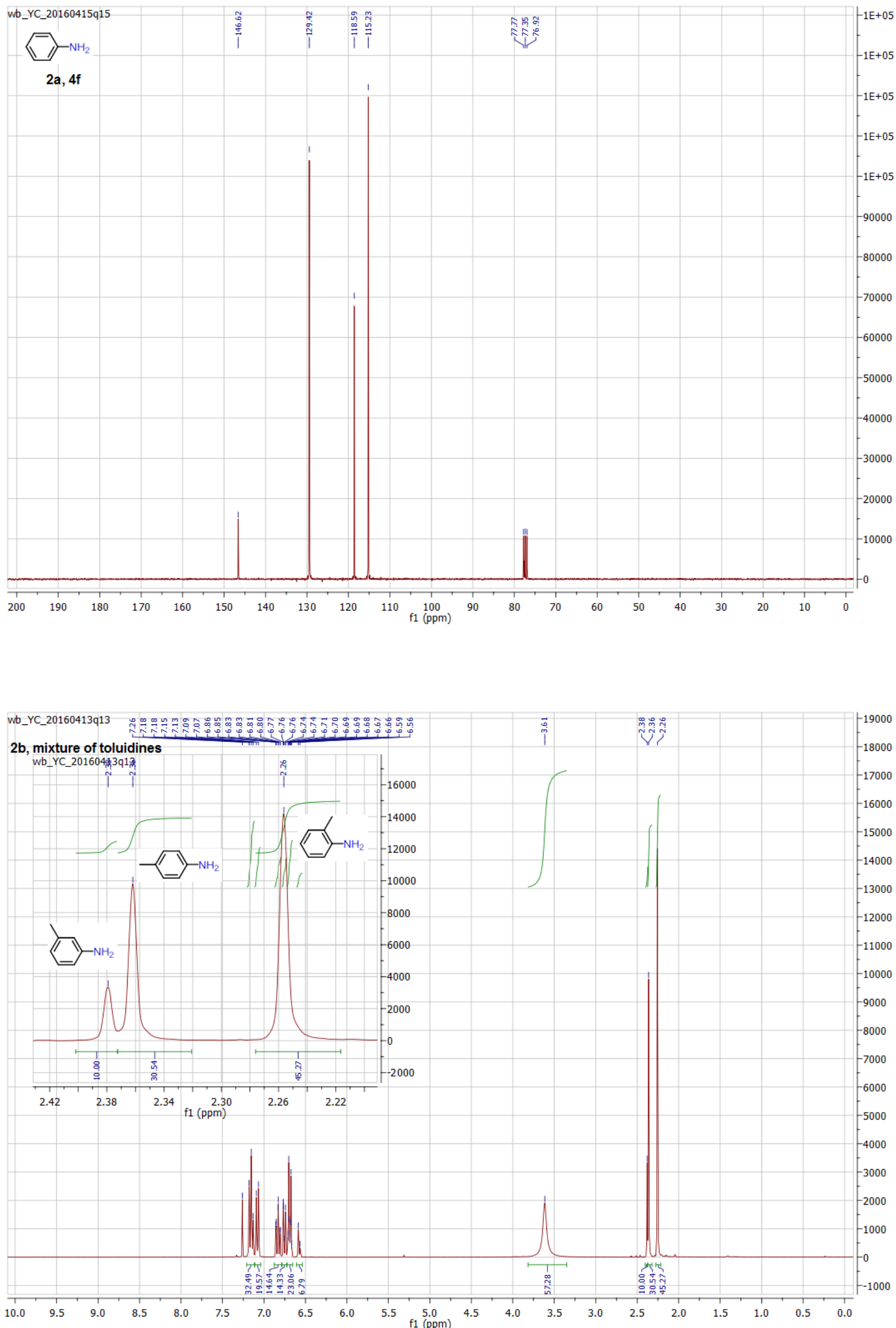

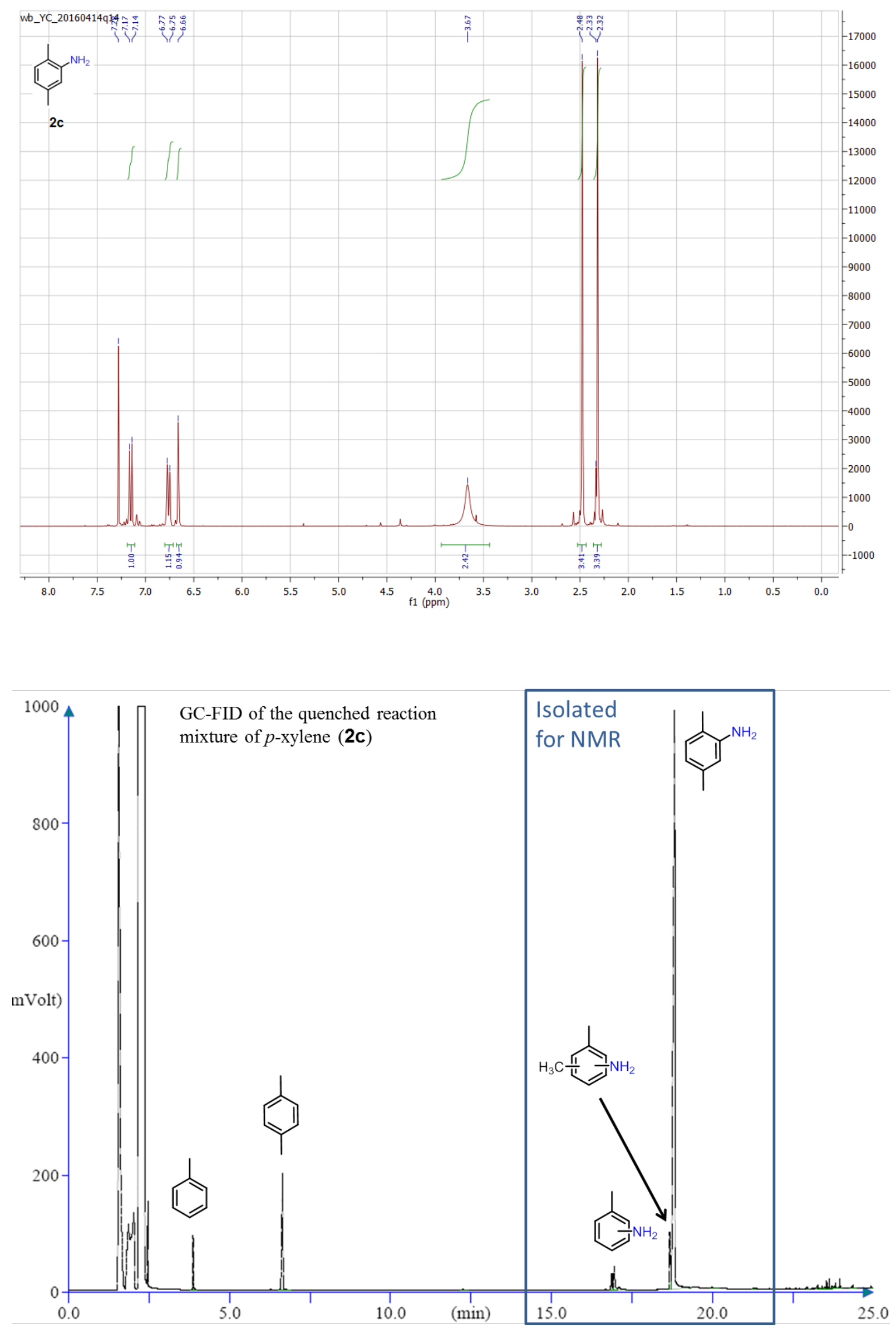

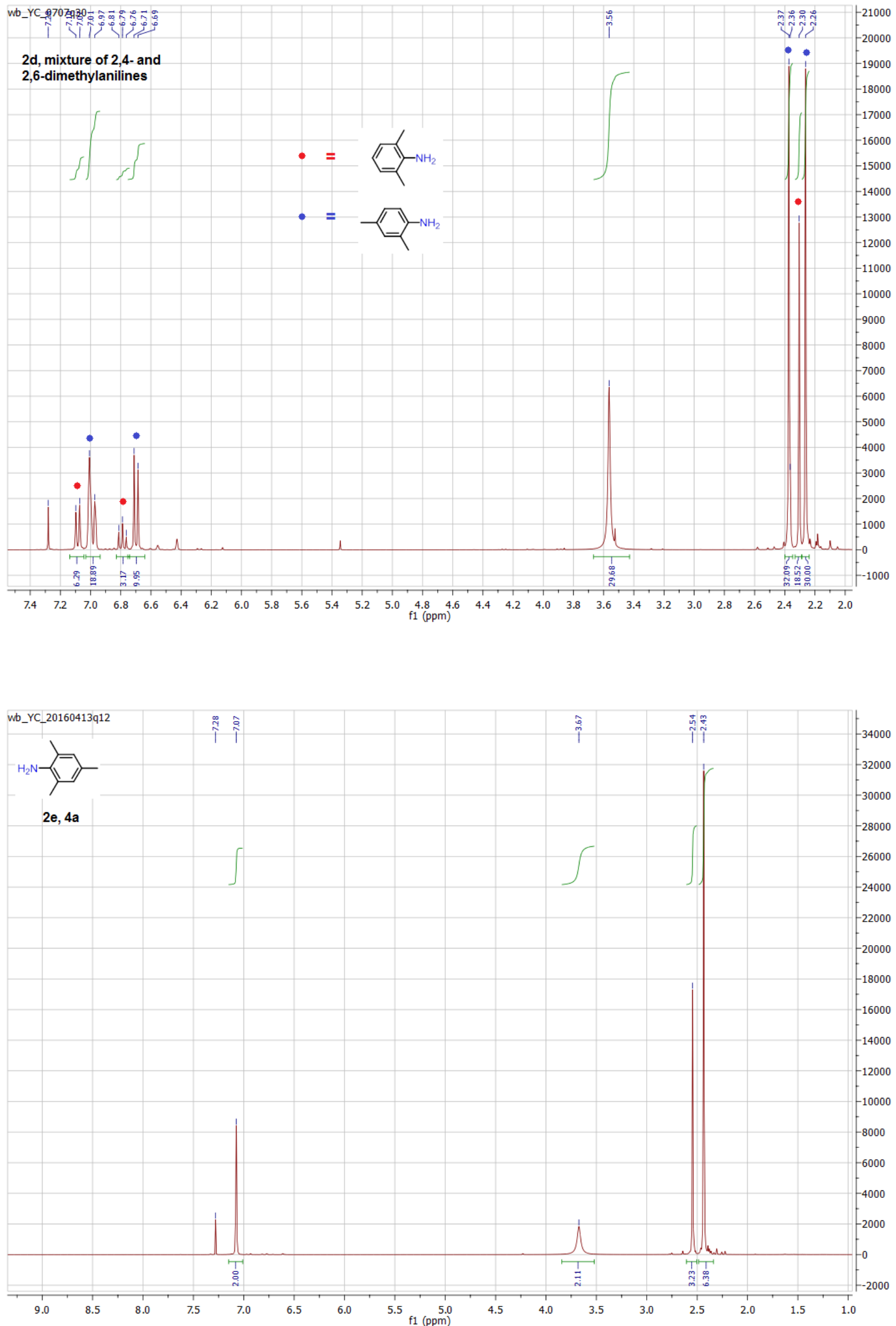

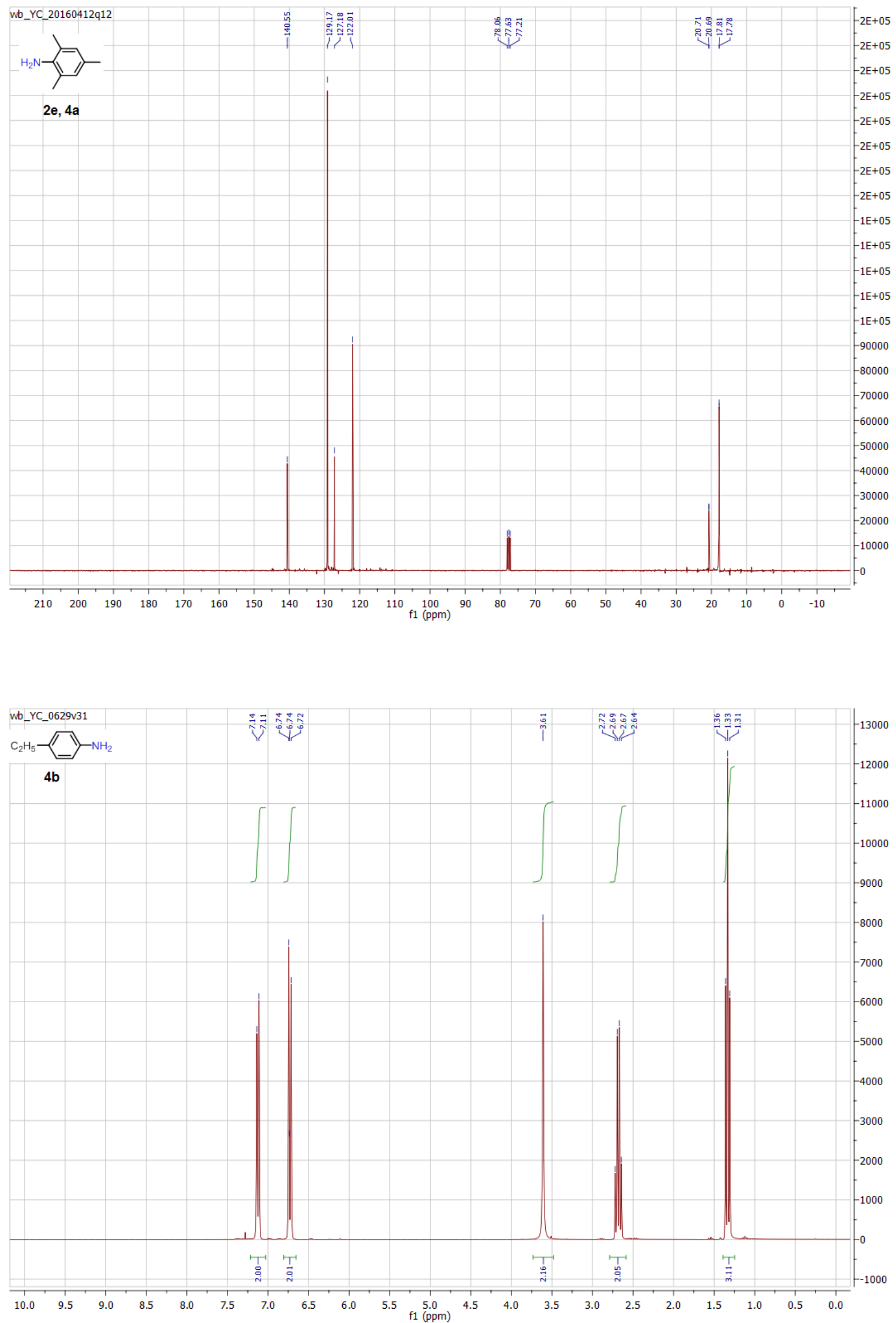

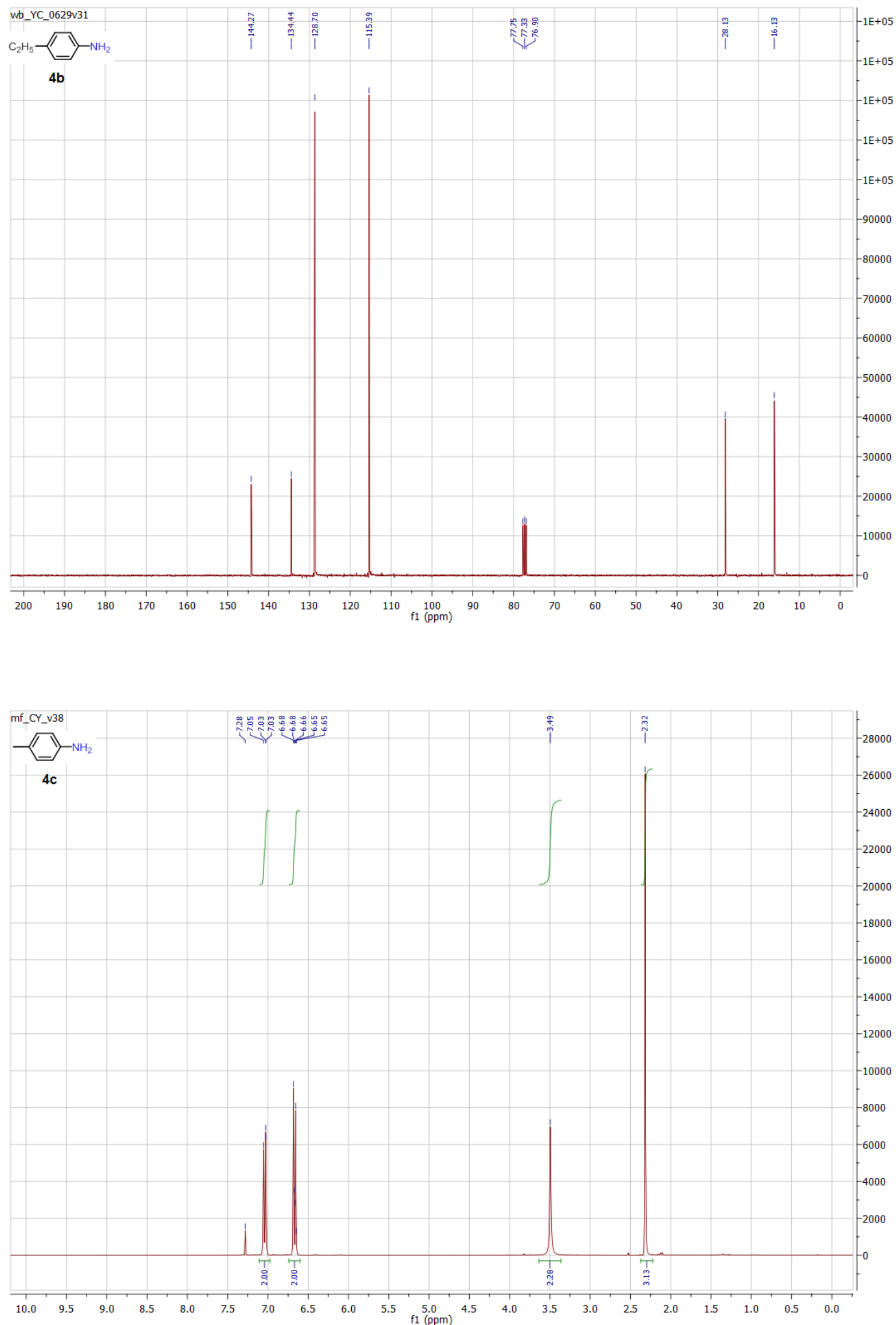

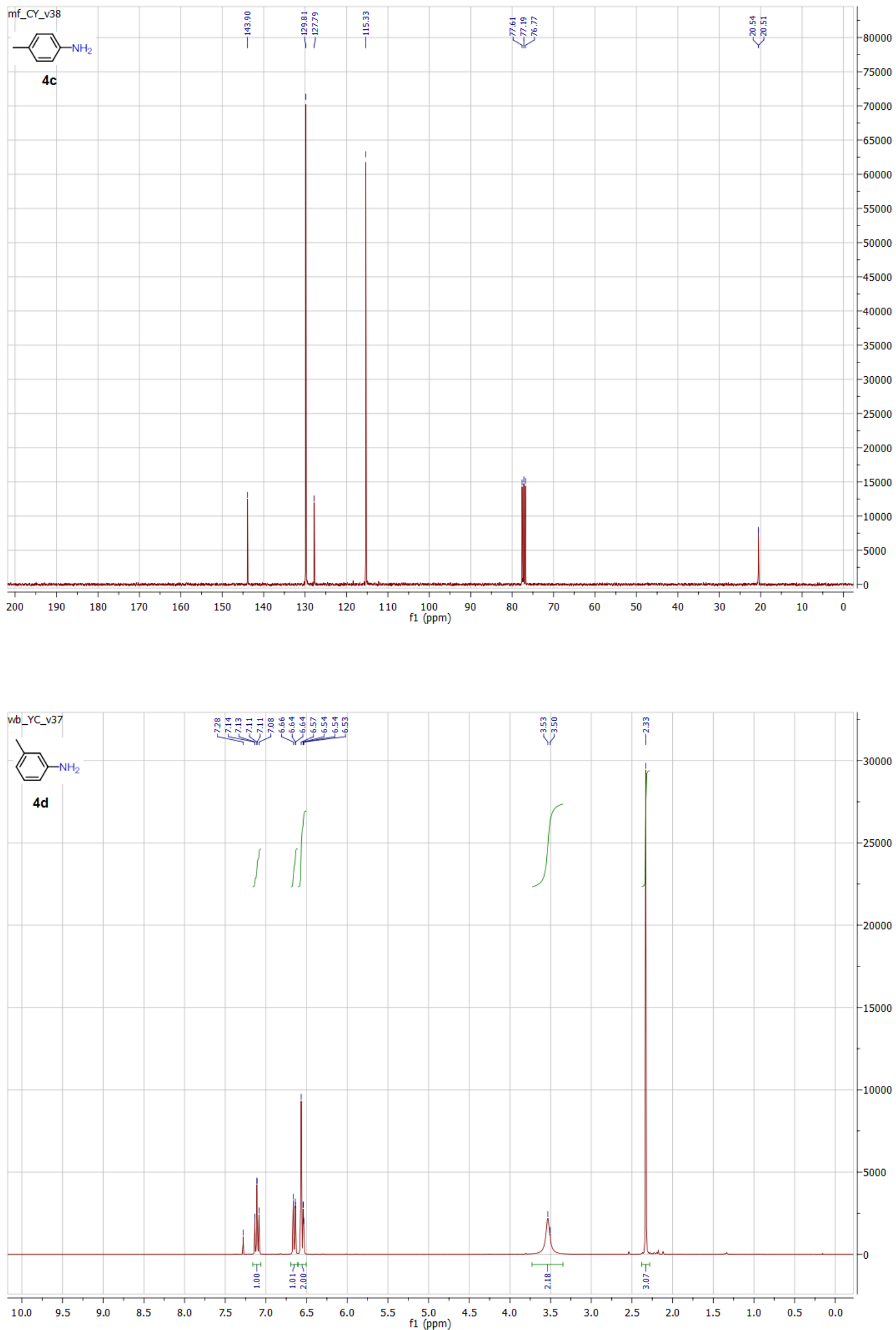

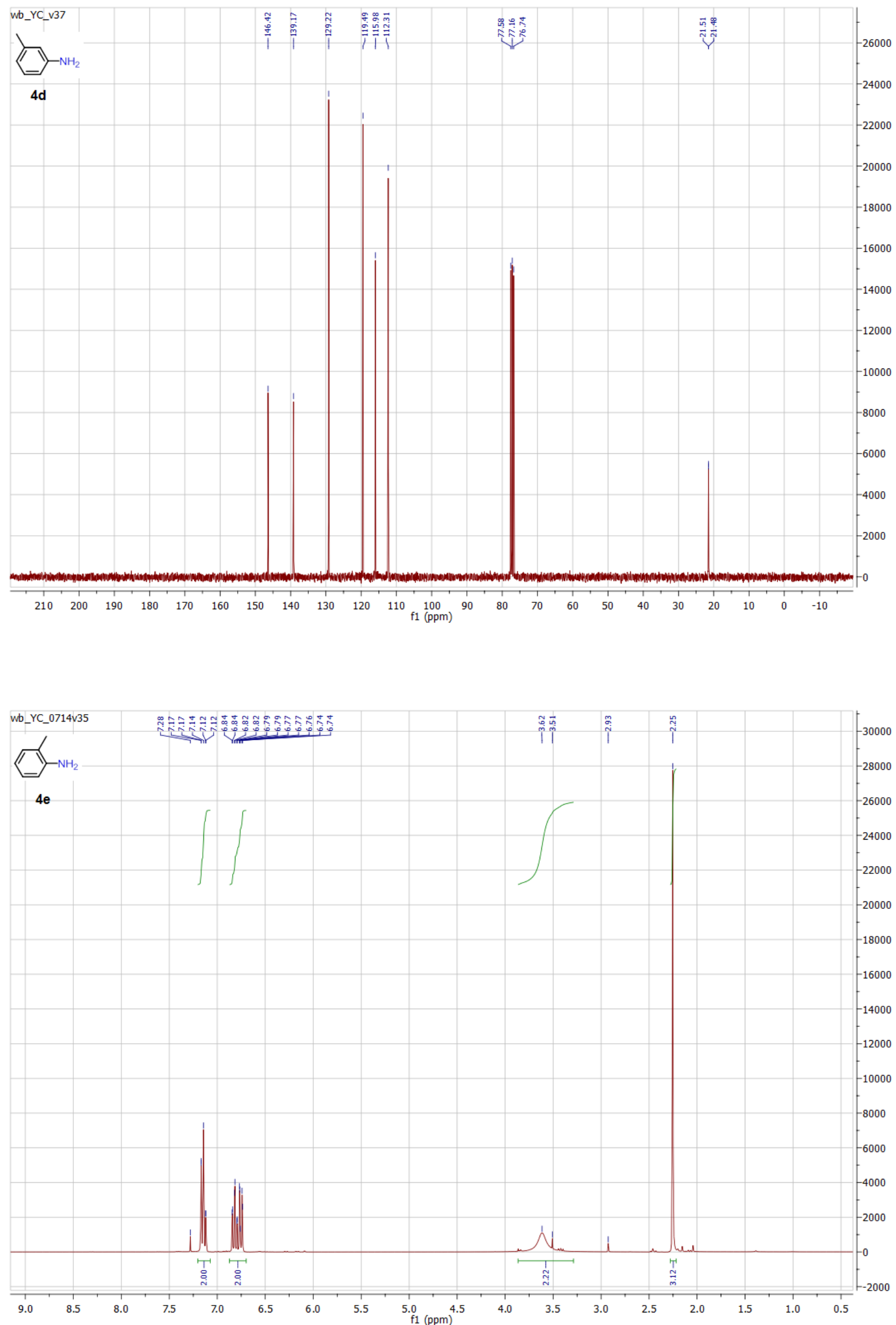

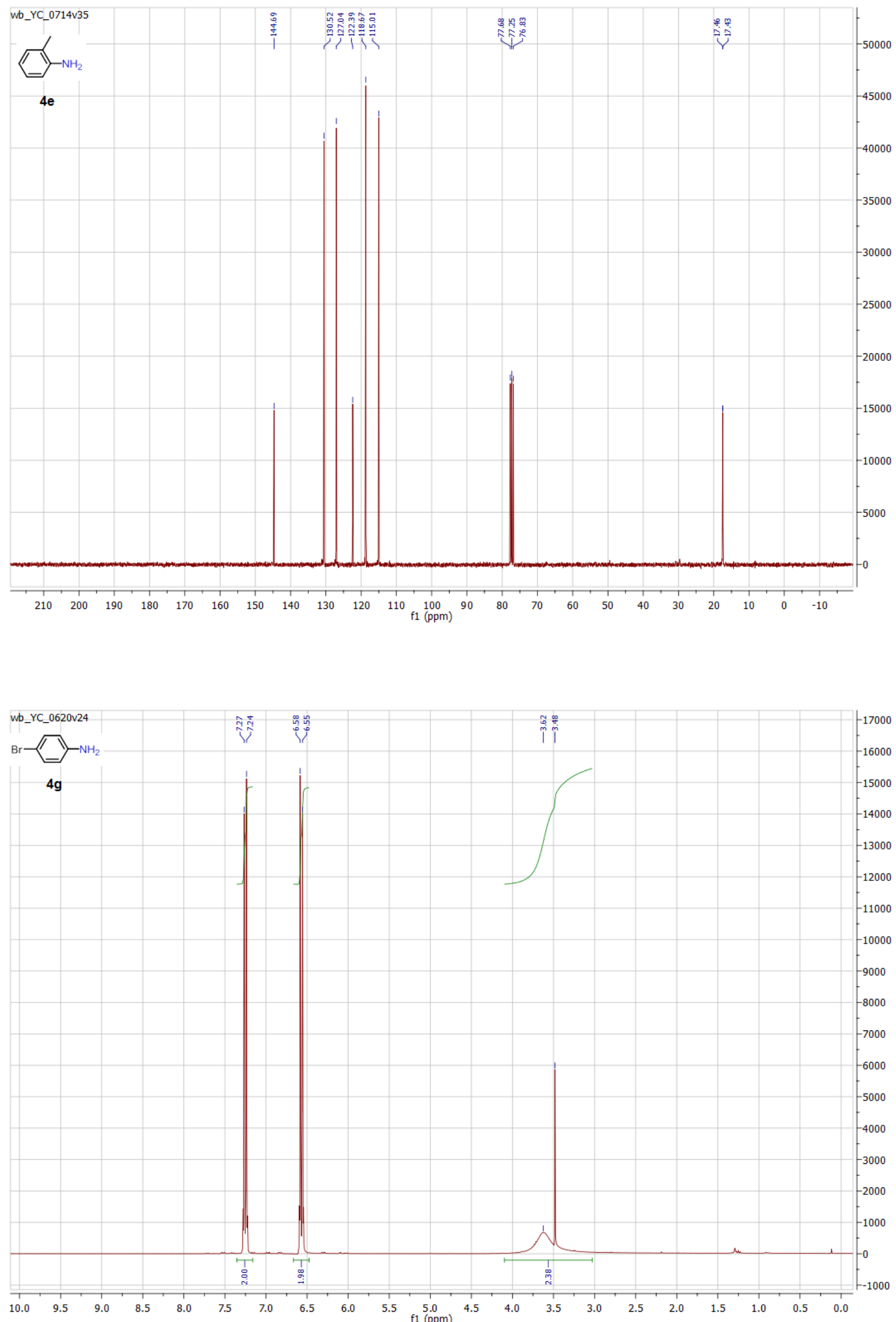

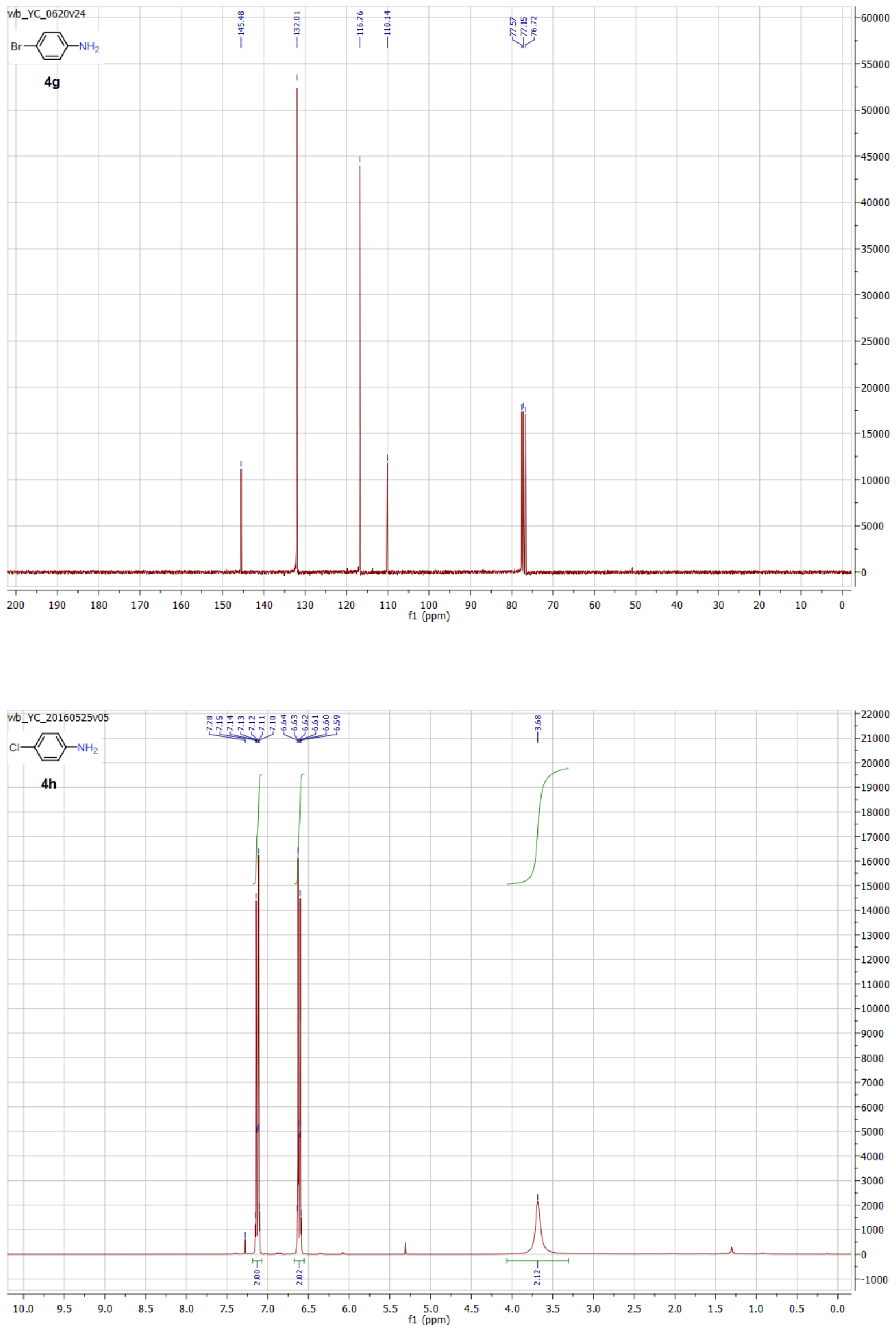

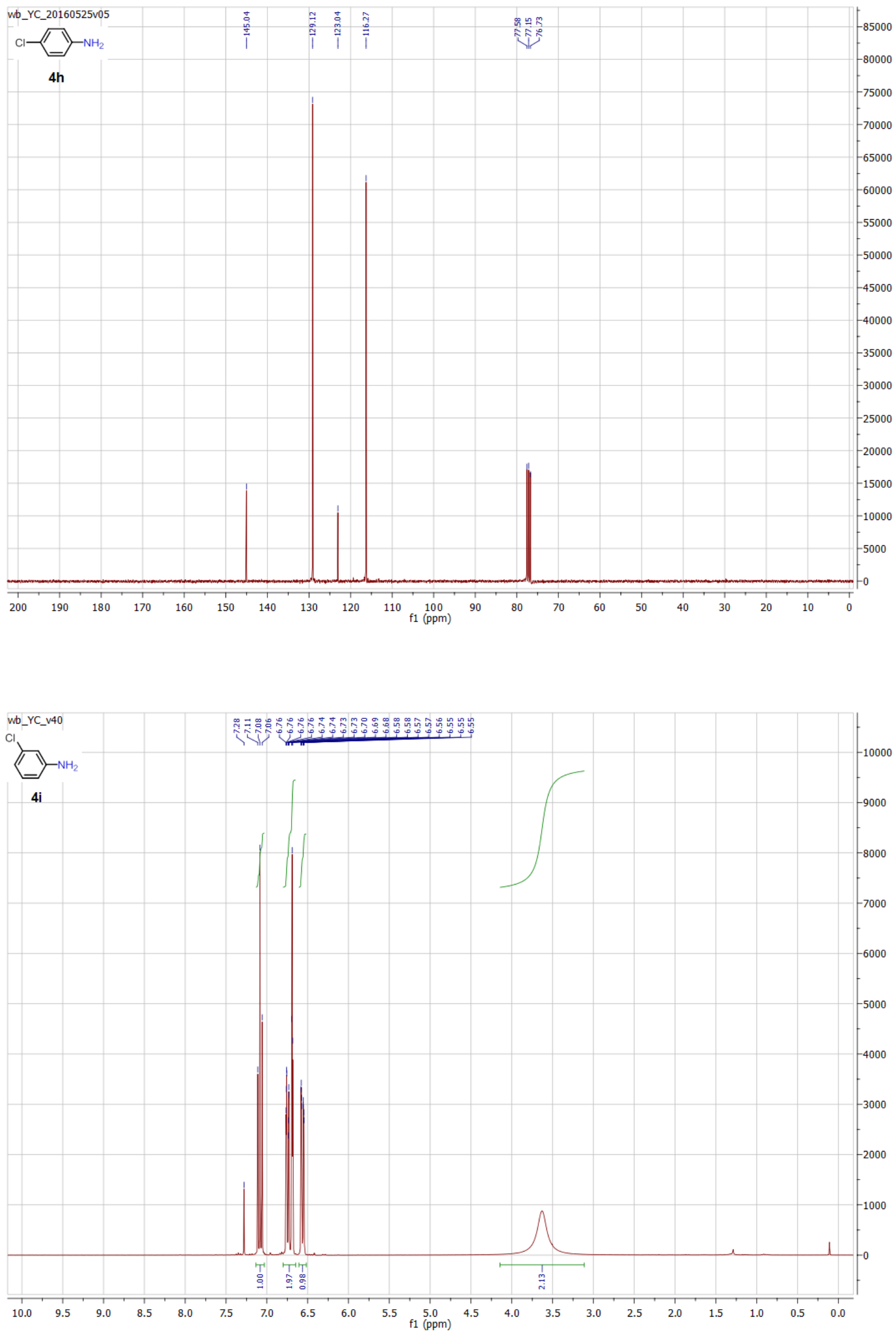

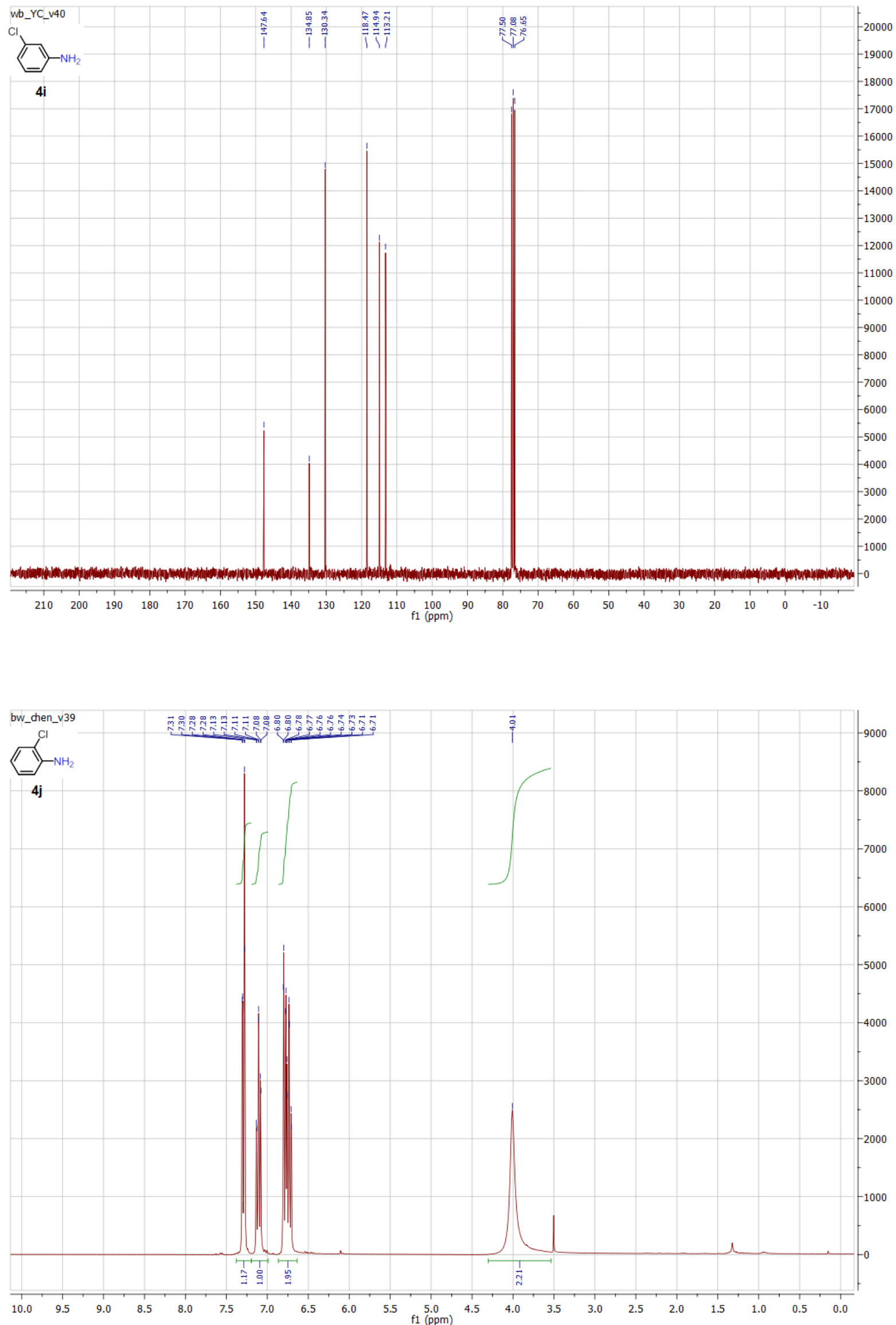

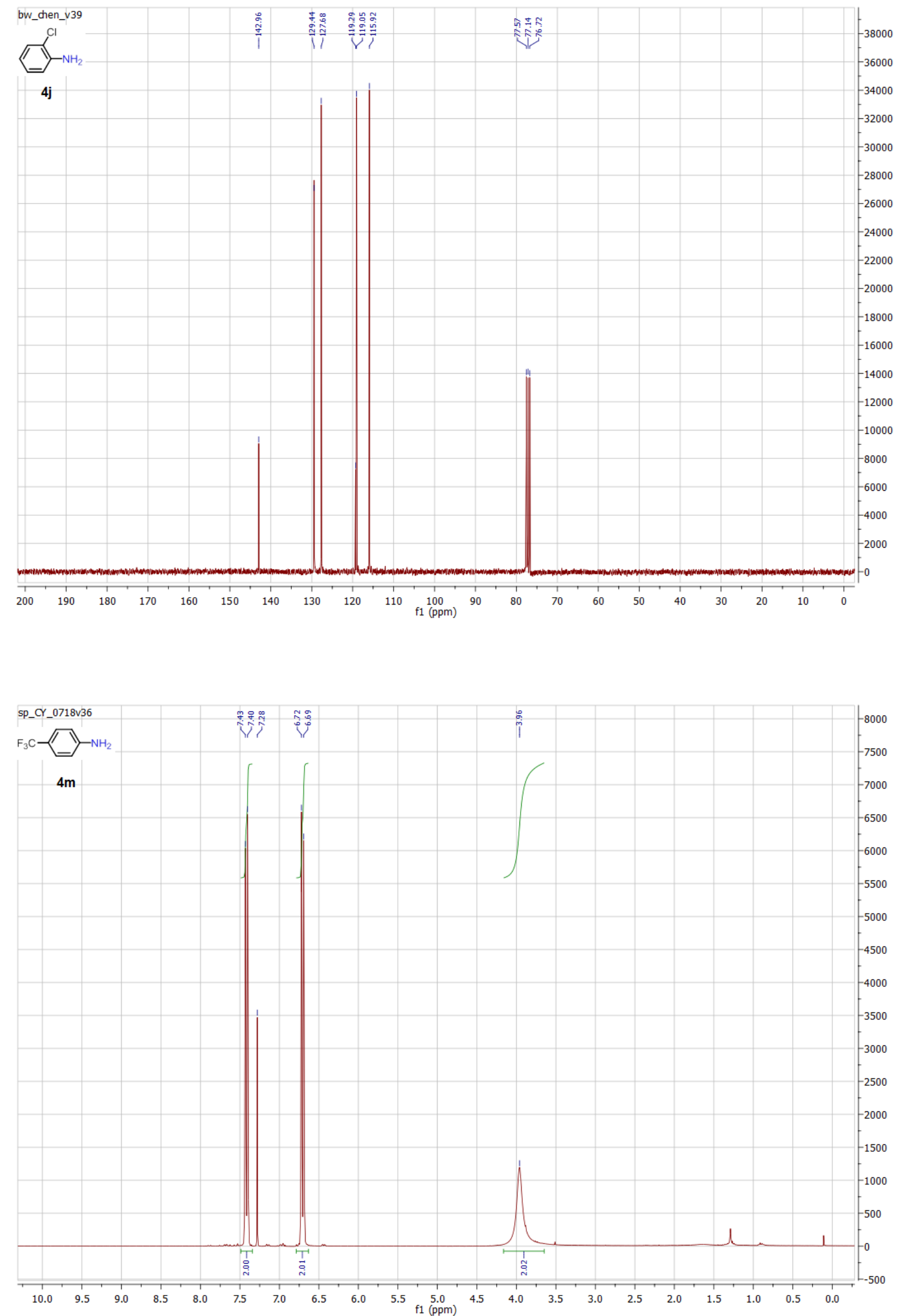


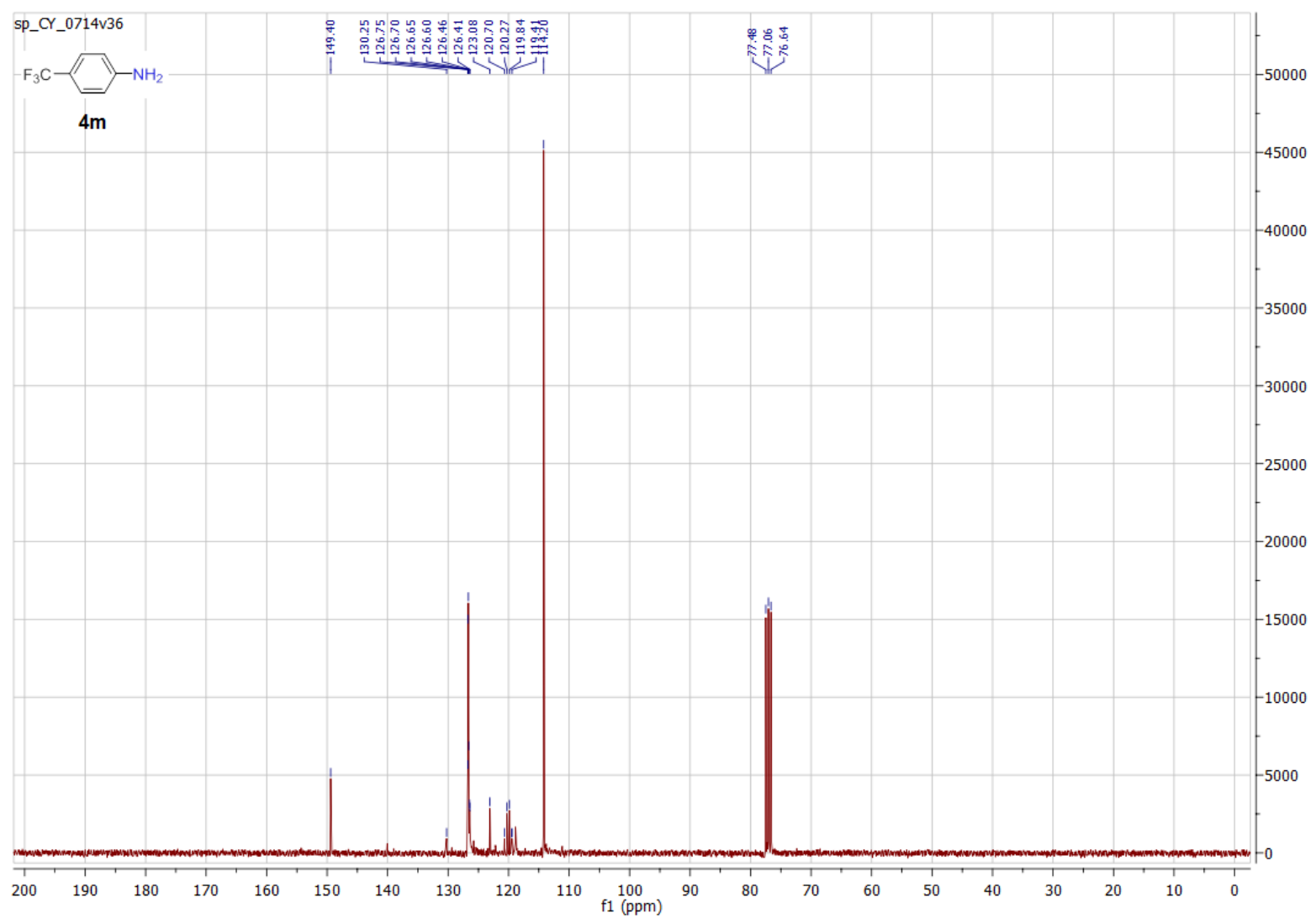

